\title{
A Novel Model of Acute Myocardial Ischemia in Human Ventricular Cardiomyocytes
}

\author{
Marta Gironés-Sangüesa ${ }^{1}$, Claudia Esteban ${ }^{1}$, Ana González-Ascaso ${ }^{1,2}$, \\ José F Rodríguez-Matas ${ }^{2}$, José M Ferrrero ${ }^{1,2}$ \\ ${ }^{1}$ CI2B, Univeristat Politècnica de València,Valencia, Spain \\ ${ }^{2}$ Chemistry, Materials and Chemical Engineering Department, Politécnico di Milano, Milano, Italy
}

\begin{abstract}
Acute myocardial ischemia is known to be a pathology with a high mortality worldwide. In the last decades, computational simulation has been a widely used tool to study the electrophysiological effects of ischemia. In this work, we present an up-to-date model of the effects of myocardial ischemia in the action potential which includes more ischemia-affected current alterations than any other previously published model. The model was used to simulate action potentials during a 10-minute period of dynamic ischemia, and the main biomarkers of the action potential were monitored, as well as the extracellular potassium elevation. The results agree with those obtained experimentally, suggesting that the model can be useful for $3 D$ simulations of acute myocardial ischemia.
\end{abstract}

\section{Introduction}

Acute myocardial ischemia (AMI) is a cardiac disease caused by the block of blood flow to the heart muscle. As a result, a set of electrophysiological alterations occurs in the tissue that increase the likelihood of ventricular arrhythmias [1].

Computational models have been widely used to study the effects of AMI on the action potential and on the propagation of the electrical activity in whole virtual hearts (see [2] for review and [3] for comparison). Most of the models incorporate changes in several ionic currents provoked by ischemia itself. For instance, ATP and ADP levels affect currents such as the ATP-sensitive potassium current [3,4], and intracellular and extracellular $\mathrm{pH}$ alter the sodium and calcium inward currents [4,5]. Changes in these (and other) parameters also affect other ionic currents, but these alterations are often ignored in previous ischemic action potential models. Moreover, the kinetics of the ischemia-affected channels is often unchanged, and only the maximum conductances are altered [2,3]. Also, most of them are based on ischemic information obtained from animal models and have not been tailored for human.
Moreover, the value of extracellular potassium concentration $\left(\left[\mathrm{K}^{+}\right]_{\mathrm{o}}\right)$ is usually imposed in ischemic simulations (with a high value that accounts for ischemiainduced hyperkalemia), but in reality, it changes dynamically during the ischemic period.

In this work, we propose a new model to simulate the electrophysiological effects of acute ischemia in the action potential that includes a description of the alterations induced by ischemia in a more comprehensive set of ionic currents. Whenever possible, the data were obtained from experiments on human cardiomyocytes.

\section{Methods}

We chose the O'Hara dynamic action potential model [6] as the "basal" model, as it was the most widely used one when this work began. Before incorporating ischemic effects, the fast sodium current was modified as in our previous works $[7,8]$.

Alterations caused by acute ischemia were introduced in the model by modifying and incorporating the ischemic effects in several currents. To simulate the changes in the intracellular ATP and ADP ([ATP $]_{i}$ and $[\mathrm{ADP}]_{\mathrm{i}}$, respectively) due to hypoxia, we added the ATP-sensitive $\mathrm{K}^{+}$current ( $\left.\mathrm{I}_{\mathrm{K}(\mathrm{ATP})}\right)$ proposed by Ferrero et al. [4] and we adjusted it to human myocytes by changing the maximum conductance and the sensitivity to $[\mathrm{ATP}]_{\mathrm{i}}$ and $[\mathrm{ADP}]_{\mathrm{i}}$ based on data obtained by Babenko et al. [9] in human cardiomyocytes. Furthermore, different scaling factors that depend on $[\mathrm{ATP}]_{\mathrm{i}}$ and $[\mathrm{ADP}]_{\mathrm{i}}$ were introduced in the formulations of the $\mathrm{Na}^{+} / \mathrm{K}^{+}$, sarcolemmal $\mathrm{Ca}^{2+}$ and SERCA pumps ( $\mathrm{I}_{\mathrm{NaK}}, \mathrm{I}_{\mathrm{pCa}}$ and $\mathrm{I}_{\mathrm{up}}$, respectively), as in Cortassa et al. [10]. Intracellular and extracellular acidosis $\left(\mathrm{pH}_{\mathrm{i}}, \mathrm{pH}_{\mathrm{o}}\right.$, respectively) were incorporated in the model in $\mathrm{I}_{\mathrm{NaK}}$, the sodium-calcium exchanger $\left(\mathrm{I}_{\mathrm{NaCa}}\right)$, the SERCA pump and the calcium release current in the sarcoplasmic reticulum ( $\left.\mathrm{I}_{\mathrm{rel}}\right)$. We also introduced changes exerted by $\mathrm{pH}_{\mathrm{i}}, \mathrm{pH}_{\mathrm{o}}$ in the L-type $\mathrm{Ca}^{2+}$ current $\left(\mathrm{I}_{\mathrm{Ca}(\mathrm{L})}\right)$ by reformulating its activation and inactivation gates and adding a scaling factor in the equation of $\mathrm{I}_{\mathrm{CaL}}$ to mimic the experiments by Saegusa et al. [11]. Also, the fast and late $\mathrm{Na}^{+}$currents ( $\mathrm{I}_{\mathrm{Na}}$ 
and $\mathrm{I}_{\mathrm{NaL}}$, respectively) and the $\mathrm{Na}^{+} / \mathrm{K}^{+}$pump were multiplied by different scaling factors that depend on extracellular and intracellular $\mathrm{pH} \quad\left(\mathrm{pH}_{\mathrm{o}}\right.$ and $\mathrm{pH}_{\mathrm{i}}$, respectively), and intracellular LPC concentration ([LPC $]_{\mathrm{i}}$ ) [12]. The rapid potassium current $\left(\mathrm{I}_{\mathrm{Kr}}\right)$ was also modified to account for the effects of LPC [13] and $\mathrm{pH}_{\mathrm{o}}$. Finally, the effects of $\mathrm{pH}_{\mathrm{o}}$ on the transient outward current $\left(\mathrm{I}_{\mathrm{to}}\right)$ were also introduced in the model [14].

The ischemic-related parameters $\left([\mathrm{ATP}]_{\mathrm{i}},[\mathrm{ADP}]_{\mathrm{i}}, \mathrm{pH}_{\mathrm{i}}\right.$, $\mathrm{pH}_{\mathrm{o}},[\mathrm{LPC}]_{\mathrm{i}}$ ) were varied dynamically as in [8] to mimic their behavior in the first 10 minutes of ischemia.

On the other hand, the model used in the simulations considers the following compartments: the intracellular space, the interstitial extracellular clefts and a bulk extracellular medium in which concentrations were assumed to be constant. The following equation simulates the dynamic changes in $\left[\mathrm{K}^{+}\right]_{\mathrm{o}}[8]$ :

$$
\frac{d\left[K^{+}\right]_{o}}{d t}=-\frac{A_{m}}{V_{o} \cdot F} I_{K, t o t}-\frac{\left[K^{+}\right]_{o}-\left[K^{+}\right]_{b u l k}}{\tau_{d i f f}}
$$

where $\mathrm{F}$ is the Faraday constant, $V_{o}$ is the extracellular volume, $A_{m}$ the area of the myocyte and $I_{K, t o t}$ is the total ionic current related to potassium. In the second term, $\tau_{\text {diff }}$ in the time constant for the diffusion of potassium and $\left[K^{+}\right]_{o}$ and $\left[K^{+}\right]_{b u l k}$ are the potassium concentrations in the extracellular space and in the bulk, respectively [8].

In each simulation, the cell was paced at a specific basic cycle length (BCL) during 5 minutes of normoxia (minute -5 to minute 0 ) in order for the virtual cell to reach a steadystate. At the 0 mark, dynamic ischemia began to develop and the cell was paced with the same BCL for 10 minutes (minute 0 to minute 10 ).

\section{Results}

Figure 1 shows simulated action potentials (two consecutive ones) at minutes $0,2.5$ and 5 after the onset of ischemia. The cell was paced at a basic cycle length (BCL) of 1,000 milliseconds ( 60 beats per minute). At the 2.5 minute mark, action potentials have shortened and their upstroke velocity has decreased. At the 5 minute mark, alternans appear. The "long" action potentials have an upstroke divided into two phases, while the "short" ones lack the second phase. This results closely resemble those obtained experimentally in animals [18]. To our knowledge, there are no true action potentials experimentally recorded in acutely ischemic human hearts to compare with.

Three preclinical biomarkers of the action potential (namely, peak membrane potential $\mathrm{V}_{\max }$, resting membrane potential RMP and action potential duration at $90 \%$ repolarization $\mathrm{APD}_{90}$ ) were continuously monitored, along with $\left[\mathrm{K}^{+}\right]_{\mathrm{o}}$, during the 10 minute ischemic period. Figure 2 shows the time course of said parameters during the simulation (with a BCL of 1,000 milliseconds). The figure shows that RMP increases (adopting less negative values) and then plateaus at a value of -62 millivolts, closely following the time course of $\left[\mathrm{K}^{+}\right]_{\mathrm{o}}$. The peak membrane potential slightly declines at the beginning of the ischemic period, shows a bifurcative behavior during the alternating period of the action potential, and collapses to a low value during the $\left[\mathrm{K}^{+}\right]_{\mathrm{o}}$ plateau.

The middle panel clearly shows the expected decrease in $\mathrm{APD}_{90}$ (caused mainly by the progressive $\mathrm{I}_{\mathrm{K}(\mathrm{ATP})}$ activation), followed by the alternating period (which lasts for approximately 2 minutes, after which $\mathrm{APD}_{90}$ continues decreasing. Finally, $\left[\mathrm{K}^{+}\right]_{0}$ exhibits its well-known biphasic time course [19], with an initial increase followed by a plateau at approximately $12 \mathrm{mmol} / \mathrm{L}$.

The results depicted in the previous figure must be compared to experimental results in human hearts in order to assess the validity of the model in reproducing human myocardial ischemic changes in the action potential. The experimental data published in this respect is scarce. Sutton et al. [20] recorded monophasic action potentials (MAPs) in human in-vivo hearts of patients undergoing coronary artery surgery. They reported a decrease in MAPD $_{90}$ of 67 milliseconds after 3 minutes of myocardial ischemia. For the same BCL (500 milliseconds), our simulation yields a decrease of 68 milliseconds.

As for the increase in $\left[\mathrm{K}^{+}\right]_{\mathrm{o}}$, experimental data in humans is non-existent. However, a study by Kazbanov et al. [21], based on clinical/experimental data from Bradley et al. [22] in human in-vivo hearts of patients during myocardial ischemia with ventricular fibrillation, indirectly estimates the rise in $\left[\mathrm{K}^{+}\right]_{\mathrm{o}}$ during the first 3 minutes of acute ischemia. They report an average increase of $2.2 \pm 1.2 \mathrm{mmol} / \mathrm{L}$, while our model slightly overestimates the rise in $\left[\mathrm{K}^{+}\right]_{\mathrm{o}}$ yielding $3.2 \mathrm{mmol} / \mathrm{L}$ in similar conditions.

\section{Conclusion}

A new and more comprehensive model for acute myocardial ischemia has been developed based on the O'Hara-Rudy model by incorporating new ischemic effects on the ionic currents. The results obtained at the cellular level regarding action potential duration and extracellular potassium accumulation are in accordance with experimental data recorded or estimated in in-vivo human hearts.

\section{Acknowledgments}

This work was partially supported by the "Programa Salvador de Madariaga 2018" of the Spanish Ministry of Science, Innovation and Universities (Grant Reference PRX18/00489). 


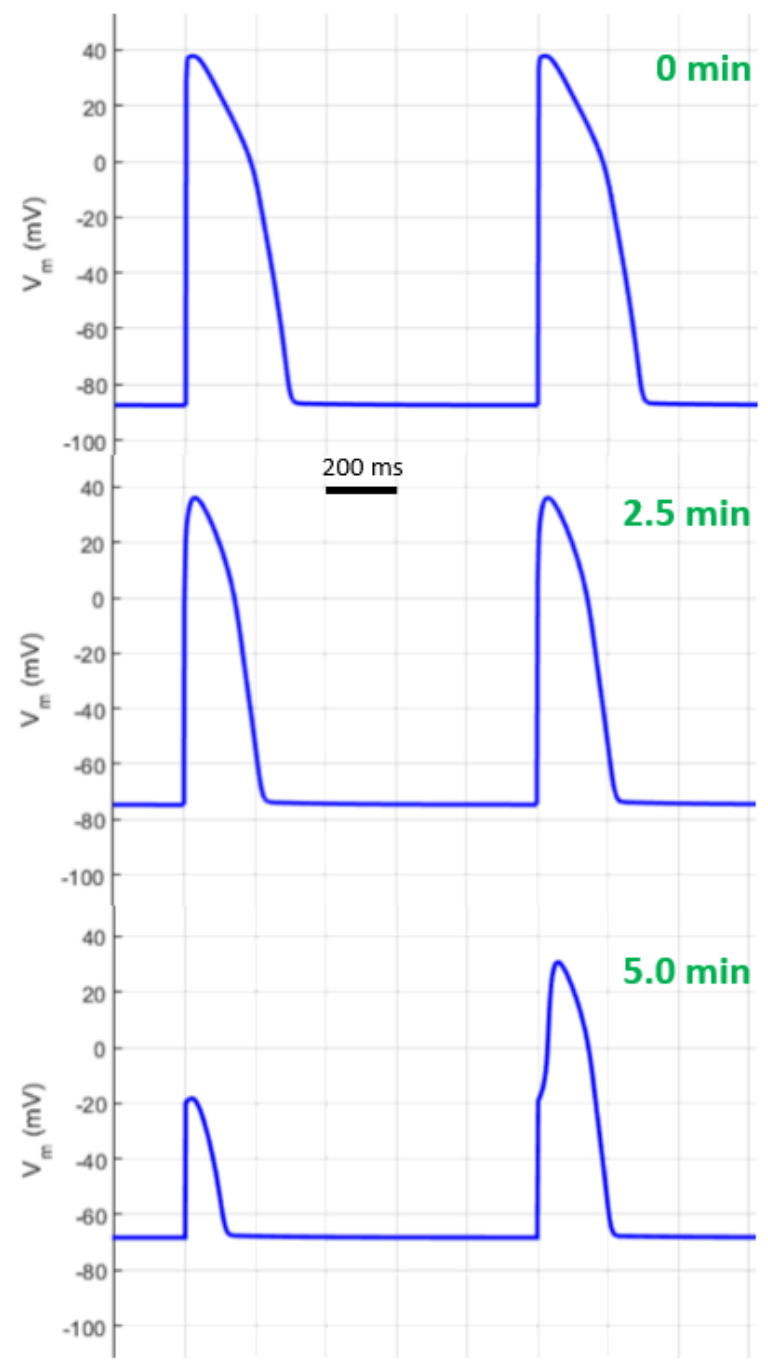

Figure 1. Action potentials (two consecutive ones) $0,2.5$ and 5 minutes after the initiation of simulated ischemia.
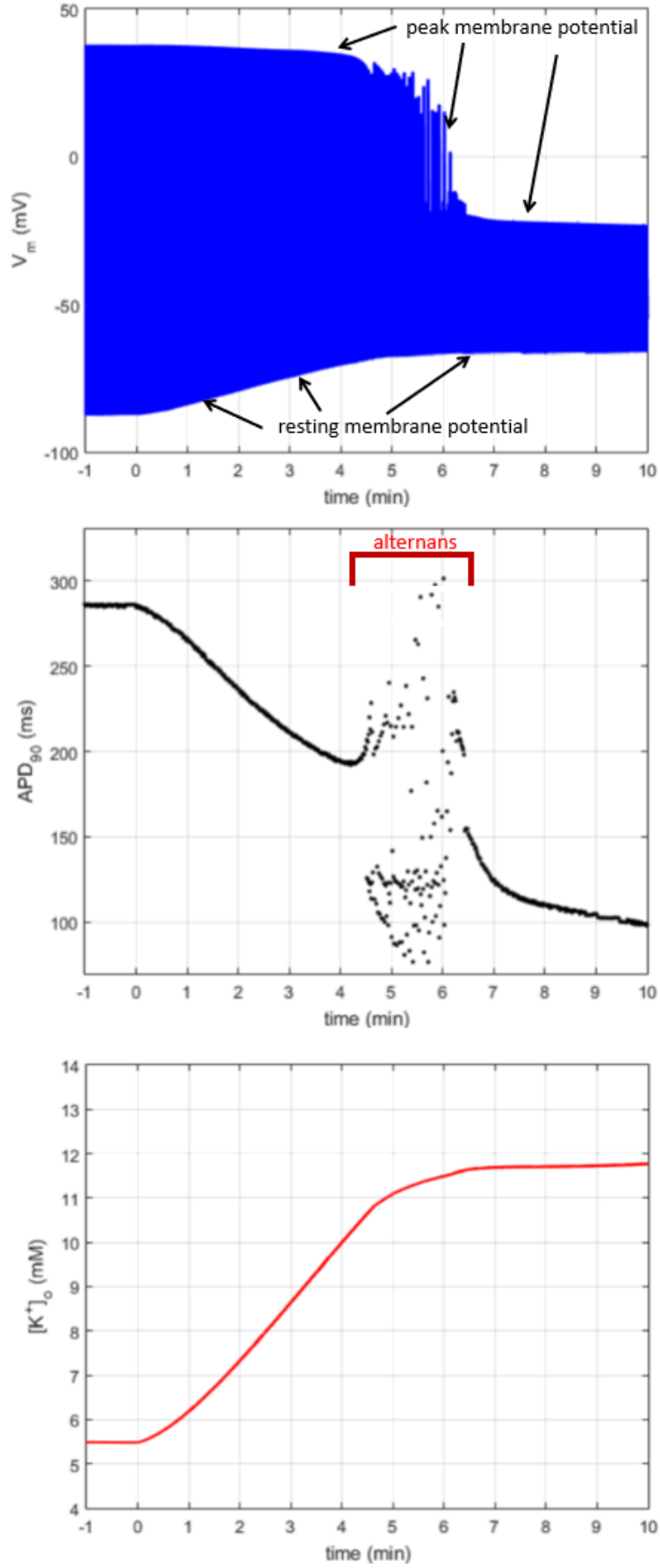

Figure 2. Upper panel: time-course of action potential (with indication of the peak membrane potential and the resting membrane potential) during the last minute of normoxia and the first 10 minutes of simulated acute myocardial ischemia. Middle panel: time course of action potential duration $\left(\mathrm{APD}_{90}\right)$, showing the alternating period. Lower panel: time course of extracellular potassium concentration. 


\section{References}

[1] M. J. Janse and A. G. Kléber, "Electrophysiological changes and ventricular arrhythmias in the early phase of regional myocardial ischemia.," Circ. Res., vol. 49, no. 5, pp. 10691081, Nov. 1981.

[2] J.M. Ferrero, B. Trénor, L. Romero. Multiscale computational analysis of the bioelectric conse-quences of myocardial ischaemia and infarction. Europace. 2014; 16: $405-15$.

[3] S. Dutta, A. Mincholé, T.A. Quinn, B. Rodriguez. Electrophysiological properties of computational human ventricular cell action potential models under acute ischemic conditions. Prog Biophys Mol Biol. 2017 Oct;129:40-52.

[4] J.M. Ferrero, J. Sáiz, J.M. Ferrero, and N.V. Thakor, "Simulation of Action Potentials From Metabolically Impaired Cardiac Myocytes," Circ. Res., vol. 79, no. 2, pp. 208-221, Aug. 1996.

[5] J.M. Ferrero, B. Trénor, B. Rodríguez, J. Saiz. Electrical activity and reentry during acute regional myocardial ischemia: insights from simulations. Int J Bifurc Chaos. 2003; $13: 3703-15$.

[6] T. O'Hara, L. Virág, A. Varró, and Y. Rudy, "Simulation of the Undiseased Human Cardiac Ventricular Action Potential: Model Formulation and Experimental Validation," PLoS Comput. Biol., vol. 7, no. 5, p. e1002061, May 2011.

[7] E. F. Carpio et al., "Optimization of lead placement in the right ventricle during cardiac resynchronization therapy. A simulation study," Front. Physiol., vol. 10, p. 74, Feb. 2019.

[8] A. González-Ascaso, P. Olcina, M. Garcia-Daras, J.F. Rodriguez-Matas, J.M. Ferrero, "Why Does Extracellular Potassium Rise in Acute Ischemia? Insights from Computational Simulations" in Computers in Cardiology, 2019, pp. 239-242.

[9] A.P. Babenko, G. Gonzalez, L. Aguilar-Bryan, and J. Bryan, "Reconstituted Human Cardiac K AтP Channels," Circ. Res. vol. 83, no. 11, pp. 1132-1143, Nov. 1998.

[10] S. Cortassa et al., "A computational model integrating electrophysiology, contraction, and mitochondrial bioenergetics in the ventricular myocyte," Biophys. J., vol. 91, no. 4, pp. 1564-1589, Aug. 2006.

[11] N. Saegusa, E. Moorhouse, R. D. Vaughan-Jones, and K. W. Spitzer, "Influence of $\mathrm{pH}$ on $\mathrm{Ca}^{2+}$ current and its control of electrical and $\mathrm{Ca}^{2+}$ signaling in ventricular myocytes," $J$. Gen. Physiol., vol. 138, no. 5, pp. 537-559, Nov. 2011.

[12] M. Gautier, H. Zhang and I. M. Fearon, "Peroxynitrite formation mediates LPC-induced augmentation of cardiac late sodium currents," J. Mol. Cell. Cardiol., vol. 42, no.2, pp. 241-251, Sep. 2007.

[13] Y. Bai, J. Wang, Y. Lu, H. Shan, B. Yang, Z. Wang. Phospholipid lysophosphatidylcholine as a metabolic trigger and HERG as an ionic pathway for extracellular $\mathrm{K}$ accumulation and "short QT syndrome" in acute myocardial ischemia. Cell Physiol Biochem. 2007;20(5):417-28.
[14] M. Stengl, E. Carmeliet, K. Mubagwa, W. Flameng. Modulation of transient outward current by extracellular protons and $\mathrm{Cd}^{2+}$ in rat and human ventricular myocytes. $J$ Physiol. 1998 Sep 15;511 ( Pt 3)(Pt 3):827-36.

[15] K. Sakamoto, M. Ishikawa, K. Koga, T. Urshidani and T. Nagao, "Energy preserving effect of 1-cis diltiazem in isolated ischemic and Reperfused guinea pig hearts: A 31PNMR Study,” Jpn. J. Pharmacol., vol. 83, no. 3, pp. 225232, Jul. 2000.

[16] P. Deleau, "Lysophosphatidylcholine, a metabolite which accumulates early in myocardium during ischemia, reduces gap junctional coupling in cardiac cells," J. Mol. Cell. Cardiol., vol. 31, no.7, pp. 1391-1401, Jul. 1999.

[17] J. R. Terkildsen, E. J. Crampin and N. P. Smith, "The balance between inactivation and activation of the $\mathrm{Na}+\mathrm{K}+$ pump underlies the triphasic accumulation of extracellular $\mathrm{K}+$ during myocardial ischemia," Am. J. Physiol. Heart Circ. Physiol., vol. 293, no. 5, pp. H3036-3045, Nov. 2007.

[18] A.G. Kléber, M.J. Janse, F.J. van Capelle, D. Durrer. Mechanism and time course of S-T and T-Q segment changes during acute regional myocardial ischemia in the pig heart determined by extracellular and intracellular recordings. Circ Res. 1978 May;42(5):603-13.

[19] A. A. Wilde and G. Aksnes, "Myocardial potassium loss and cell depolarisation in ischemia and hypoxia," Cardiovasc. Res., vol. 29, pp. 1-15, Jan. 1995.

[20] P.M. Sutton, P. Taggart, T. Opthof, R. Coronel, R. Trimlett, W. Pugsley, P. Kallis. Repolarisation and refractoriness during early ischaemia in humans. Heart. 2000 Oct;84(4):365-9.

[21] I.V. Kazbanov, R.H. Clayton, M.P. Nash, C.P. Bradley, D.J. Paterson, M.P. Hayward, P. Taggart, A.V. Panfilov. Effect of global cardiac ischemia on human ventricular fibrillation: insights from a multi-scale mechanistic model of the human heart. PLoS Comput Biol. 2014 Nov 6;10(11):e1003891.

[22] C.P. Bradley, R.H. Clayton, M.P. Nash, A. Mourad, M. Hayward, D.J. Paterson, P. Taggart. Human ventricular fibrillation during global ischemia and reperfusion: paradoxical changes in activation rate and wavefront complexity. Circ Arrhythm Electrophysiol. 2011 Oct;4(5):684-91.

Address for correspondence:

José M. Ferrero

Universitat Politècnica de València Centro de Investigación e Innovación en Bioingeniería Camino de Vera s/n, Edificio 8B cferrero@eln.upv.es 